\title{
Effects of amiodarone on short QT syndrome variant 3 in human ventricles: a simulation study
}

\author{
Cunjin Luo ${ }^{1}$, Kuanquan Wang ${ }^{1 *}$ and Henggui Zhang ${ }^{1,2,3^{*}}$
}

*Correspondence:
wangkq@hit.edu.cn;
H.Zhang-3@manchester.ac.uk
1 School of Computer
Science and Technology,
Harbin Institute
of Technology (HIT),
Harbin 150001, China
Full list of author information
is available at the end of the
article

${ }^{*}$ Correspondence:

wangkq@hit.edu.cn

1 School of Computer

Science and Technology,

Harbin Institute

Harbin 150001, Chin

is available at the end of the

article

\begin{abstract}
Background: Short QT syndrome (SQTS) is a newly identified clinical disorder associated with atrial and/or ventricular arrhythmias and increased risk of sudden cardiac death (SCD). The SQTS variant 3 is linked to D172N mutation to the KCNJ2 gene that causes a gain-of-function to the inward rectifier potassium channel current $\left(I_{K 1}\right)$, which shortens the ventricular action potential duration (APD) and effective refractory period (ERP). Pro-arrhythmogenic effects of SQTS have been characterized, but less is known about the possible pharmacological treatment of SQTS. Therefore, in this study, we used computational modeling to assess the effects of amiodarone, class III anti-arrhythmic agent, on human ventricular electrophysiology in SQT3.
\end{abstract}

Methods: The ten Tusscher et al. model for the human ventricular action potentials (APs) was modified to incorporate $I_{\mathrm{K} 1}$ formulations based on experimental data of Kir2.1 channels (including WT, WT-D172N and D172N conditions). The modified cell model was then implemented to construct one-dimensional (1D) and 2D tissue models. The blocking effects of amiodarone on ionic currents were modeled using $I C_{50}$ and Hill coefficient values from literatures. Effects of amiodarone on APD, ERP and pseudo-ECG traces were computed. Effects of the drug on the temporal and spatial vulnerability of ventricular tissue to genesis and maintenance of re-entry were measured, as well as on the dynamic behavior of re-entry.

Results: Amiodarone prolonged the ventricular cell APD and decreased the maximal voltage heterogeneity ( $\delta V$ ) among three difference cells types across transmural ventricular wall, leading to a decreased transmural heterogeneity of APD along a 1D model of ventricular transmural strand. Amiodarone increased cellular ERP, prolonged QT interval and decreased the T-wave amplitude. It reduced tissue's temporal susceptibility to the initiation of re-entry and increased the minimum substrate size necessary to sustain re-entry in the $2 \mathrm{D}$ tissue.

Conclusions: At the therapeutic-relevant concentration of amiodarone, the APD and ERP at the single cell level were increased significantly. The QT interval in pseudo-ECG was prolonged and the re-entry in tissue was prevented. This study provides further evidence that amiodarone may be a potential pharmacological agent for preventing arrhythmogenesis for SQT3 patients.

Keywords: Arrhythmia, Short QT syndrome, Computer simulation, KCNJ2, D172N, Amiodarone 


\section{Background}

Short QT syndrome (SQTS) is a potentially lethal cardiac ion channelopathy associated with a variety of signs and symptoms, from dizziness and fainting (syncope) to cardiac arrest and sudden death [1-6]. These signs and symptoms can occur anytime from early infancy to old age. Among which, short QT interval in SQTS patients is commonly less than $360 \mathrm{~ms}$, and T-wave morphology is tall and symmetrical. SQTS with various mutations is genetically heterogeneous, involving the KCNH2 [4], KCNQ1 [3], KCNJ2 [2], CACNA1C [7], CACNB2b [7] and CACNA2D1 [8] genes. These identified gene mutations in membrane ion channels alter the electrical activity of the heart, leading to arrhythmic characteristic of SQTS. Among the mutations, either a gain-in-function of the potassium channel or a loss-in-function of the calcium channel across the membrane of cardiac muscle cells has been observed [2-4, 7, 8].

The SQTS is associated with accelerated ventricular repolarization and with an increased risk of atrial and/or ventricular arrhythmias and of sudden cardiac death (SCD) [6, 9]. A gain-of-function $K C N J 2$ mutation has been identified in SQTS variant 3 (SQT3), with a 5-year-old child and her father exhibiting a corrected QT interval (QTc) of 315 and $320 \mathrm{~ms}$, respectively, and a narrow and peaked T-wave morphology on the clinical electrocardiogram (ECG) [2]. Based on a systematic genetic analysis, a pair of complementary bases substituted from aspartic acid to asparagine at position 172 in the Kir2.1 channel was discovered. The results from in vitro voltage clamp recordings have demonstrated an increased $I_{\mathrm{K} 1}$ in the Kir2.1 channels during terminal repolarization of the action potentials (APs) [2]. Our previous computational studies [10] have shown that SQT3 abbreviated the AP duration (APD) and effective refractory period (ERP) and steepened APD restitution (APD-R) and ERP restitution (ERP-R) curves, which consequently shortened the QT interval and raised the T-wave amplitude. Whereas pro-arrhythmogenic effects of SQT3 in the human ventricles have been investigated [2, 10], less is known about the pharmacological agents that prevent incidence of arrhythmogenesis in SQT3 patients.

Currently, the only proven therapy for SQTS patients is the application of an electric shock, delivered via an implantable cardioverter defibrillator (ICD) [9, 11-13]. Despite the success of this technique, there is an increased risk of an inappropriate shock discharged by the ICD $[9,11,12,14]$. As the QT interval is not restored within the normal range over time, ICD is unsuitable for SQTS patients [12]. Although SQTS patients may benefit from subcutaneous ICD, additional cautions are needed to affirm the safety of such device [15]. To effectively treat SQTS patients, pharmacological therapy may be the primary modality to restore the normal QT interval and protect against arrhythmia $[9$, $11,16,17]$. However, data regarding pharmacological treatment of SQTS are very limited. A clinical study by Lu et al. [18] showed that amiodarone prevented the incidence of arrhythmia and prolonged the QT interval in an SQTS patient, but electrophysiologic testing data were not available in that patient. Long-term therapy of amiodarone may be associated with unwanted effects. Important side effects were seen in the thyroid gland, liver, lung and skin. Some of these side effects are dose-dependent, and others may be related to the chemical structure and metabolism of amiodarone [19]. At present, there is no experimental model of SQT3. Moreover, drugs exert effects spanning from the ion channel to the whole organ level, making investigation using experimental techniques tough and rather expensive [20]. In recent years, computational models of the heart are 
beginning to play a critical role in the investigations of arrhythmias and anti-arrhythmic therapy. Therefore, this study was undertaken in order to assess the effects of amiodarone, a class III anti-arrhythmic agent, on human ventricular cell and tissue model in SQT3 conditions, and consequent effects of amiodarone on QT interval prolongation and prevention of re-entrant arrhythmia in this form of SQTS.

\section{Methods}

We proposed an assessment flow for evaluating the actions of amiodarone on ventricular excitation associated with SQT3 at the cell and tissue levels using mathematical models of the heart, as illustrated in Fig. 1.

\section{$I_{\mathrm{K} 1}$ kinetics and drug/ion current channel interaction}

The ten Tusscher et al. [21] model for human ventricular endocardial (ENDO), midmyocardial (MIDDLE) and epicardial (EPI) APs was used for this study. The parameters of $I_{\mathrm{K} 1}$ model were modified to incorporate the experimentally observed kinetic properties of the wild-type (WT) and mutant homozygous (D172N) and heterozygous (WTD172N) $I_{\text {Kir2.1. }}$ The $I_{\mathrm{K} 1}$ model formulation is described as

$$
I_{K 1}=G_{K 1} \sqrt{\frac{K_{o}}{5.4}} x_{K 1 \infty}\left(V-E_{K}\right)
$$

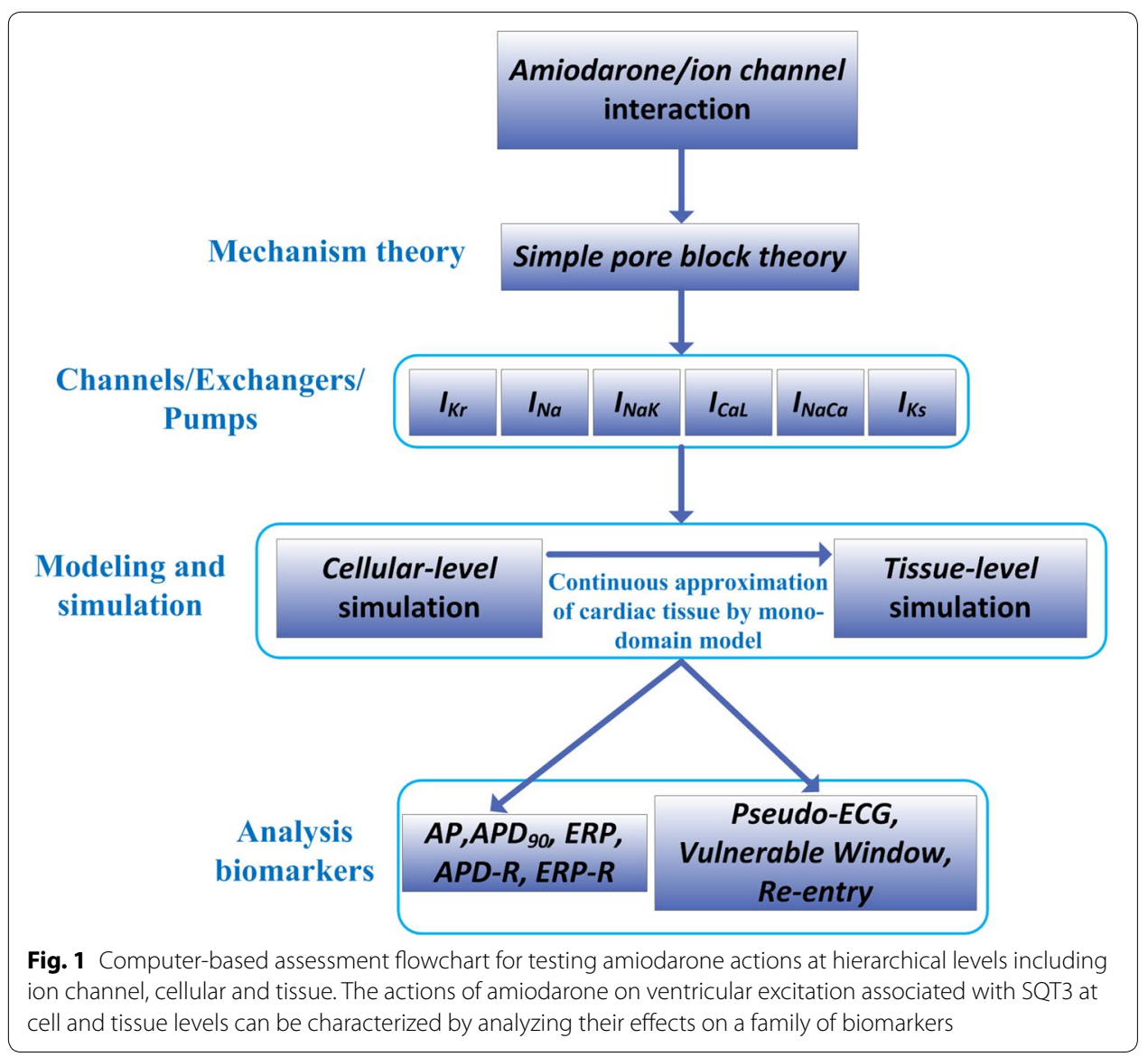


where $V$ is the transmembrane voltage, $E_{\mathrm{K}}$ is the potassium reversal potential, $G_{\mathrm{K} 1}$ is the maximum conductance, and $x_{\mathrm{k} 1 \infty}$ is a rectification factor, which is a characteristic of the open-up probability of the channel. The $I_{\mathrm{K} 1}$ model used was that of ten Tusscher [21], which was based on the formulation of Priebe and Beuckelmann [22] model. Experimentally obtained current-voltage $(I-V)$ data [23] were used to modify the $I_{\mathrm{K} 1}$ formulations for WT, WT-D172N and D172N conditions. This was achieved by simulating the experimental voltage-clamp protocol [23], with which the experimental data [23] were fitted to model equations by using the Broyden-Fletcher-Goldfarb-Shanno optimization algorithm following our previous study [10]. Relative current proportions for WT, WT-D172N and D172N conditions were scaled by using relative proportions of peak $I_{\text {Kir2.1 }}$ [23]. The peak $I_{\text {Kir2.1 }}$ was 4.6-fold for the D172N condition and 2.2-fold for the WT-D172N condition than that for the WT condition [23]. In simulations, Eq. 1 was modified to incorporate the D172N-induced changes in the channel kinetic properties, including (i) the marked augmentation of an outward Kir2.1 current through the D172N channel, and (ii) the rightward voltage shift of the peak repolarization current. The modified $I_{\mathrm{K} 1}$ formulations for WT, WT-D172N and D172N conditions [10] are shown in Additional file 1.

A simple pore block theory [24] was used in this study to model the drug/ion channel interactions. With this theory, the effect of a drug on blocking an ion channel can be simulated using a blocking factor $\theta$ that reduces the maximal conductance of an ionic current is modified in a dose-dependent manner such that:

$$
\theta=\frac{1}{1+\left(\frac{I C_{50}}{[D]}\right)^{n H}}
$$

where $[D]$ is the concentration of a drug, $\mathrm{IC}_{50}$ is the half maximal inhibitory concentration, and $\mathrm{nH}$ is the Hill coefficient. The respective reduction of ionic currents in the presence of amiodarone was determined by using $\mathrm{IC}_{50}$ and $\mathrm{nH}$ values as shown in Table 1 . In this study, two concentrations of amiodarone in the effective therapeutic range were chosen. The range of clinical therapeutic concentrations is between $1 \mu \mathrm{M}$ (low concentration) and $3 \mu \mathrm{M}$ (high concentration) [25, 26]. The resulting ion channel conductivity reductions relative to their original values in the presence of amiodarone are provided in Table 2.

Table 1 Inhibition of cardiac ion currents in the presence of amiodarone

\begin{tabular}{lllllll}
\hline Drug & \multicolumn{7}{l}{ Amiodarone } & & & & \\
\cline { 2 - 7 } Current & $\boldsymbol{I}_{\mathbf{K r}}$ & $\boldsymbol{I}_{\mathbf{N a}}$ & $\boldsymbol{I}_{\text {NaK }}$ & $\boldsymbol{I}_{\text {CaL }}$ & $\boldsymbol{I}_{\text {NaCa }}$ & $\boldsymbol{I}_{\mathbf{K s}}$ \\
\hline $\mathrm{I}_{50}(\mu \mathrm{M})$ & 2.80 & 4.84 & 15.60 & 5.80 & 3.30 & 3.84 \\
$\mathrm{nH}$ & 0.91 & 0.76 & 1.00 & 1.00 & 1.00 & 0.63 \\
Source & {$[27]$} & {$[28]$} & {$[29]$} & {$[30]$} & {$[31]$} & {$[32]$} \\
\hline
\end{tabular}

Data used in this study showing inhibition of ion currents in the presence of amiodarone. Half-maximal inhibitory concentration $\left(\mathrm{IC}_{50}\right)$ and Hill coefficient $(\mathrm{nH})$ values for amiodarone extracted from literatures 
Table 2 lon channel conductivities (\% of original value) in the presence of amiodarone

\begin{tabular}{lllllll}
\hline Conductivity & \multicolumn{7}{l}{ Amiodarone } & & & \\
\cline { 2 - 6 } & $\boldsymbol{G}_{\mathbf{K r}}$ & $\boldsymbol{G}_{\mathbf{N a}}$ & $\boldsymbol{P}_{\text {NaK }}$ & $\boldsymbol{G}_{\mathbf{C a L}}$ & $\boldsymbol{P}_{\text {NaCa }}$ & $\boldsymbol{G}_{\mathbf{K s}}$ \\
\hline Low dose $(1 \mu \mathrm{M})$ & 71.28 & 76.33 & 93.98 & 85.29 & 76.74 & 69.42 \\
High dose $(3 \mu \mathrm{M})$ & 48.41 & 58.78 & 83.87 & 65.91 & 52.38 & 53.73 \\
\hline
\end{tabular}

Ion channel conductivities (\% of original value) in the presence of amiodarone. Definition of amiodarone concentrations: low $(1 \mu \mathrm{M})$ and high $(3 \mu \mathrm{M})$

\section{Single-cell model and AP simulations}

In this study, the modified $I_{\mathrm{K} 1}$ model was incorporated into the ten Tusscher et al. [21] model for the human ventricular cell AP. In the single cell AP model, the electrophysiological activity during different phases can be described by the following equation:

$$
\frac{d V}{d t}=-\frac{I_{i o n}+I_{s t i m}}{C_{m}}
$$

where $V$ is the transmembrane voltage, $t$ is time, $C_{\mathrm{m}}$ is the cellular capacitance per unit surface area, $I_{\text {stim }}$ is the external stimulus current, and $I_{\text {ion }}$ is the sum of transmembrane ionic currents. The component of the late sodium current $\left(I_{\mathrm{NaL}}\right)$ from the ORd model [33] was incorporated. The physical units are as follows: time $t$ is in ms, potential $V$ is in $\mathrm{mV}$, current $I_{\text {ion }}$ and externally applied stimulus current $I_{\text {stim }}$ are in pA, current densities are in $\mathrm{pA} / \mathrm{pF}$, capacitance $C_{\mathrm{m}}$ is in $\mu \mathrm{F} / \mathrm{cm}^{2}$, channel conductance $G$ is in $\mathrm{nS} / \mathrm{pF}$, intraand extracellular ionic concentrations are in millimolar. The code for the cell model was downloaded from http://www-binf.bio.uu.nl/khwjtuss/SourceCodes/. The cell model was paced with an S1-S2 protocol. An S1 was applied with a basic cycle length (BCL) of $800 \mathrm{~ms}$ and an amplitude of $-52 \mathrm{pA} / \mathrm{pF}$ for $1 \mathrm{~ms}$. An S2 with the same amplitude and duration as the S1 was applied after the AP evoked by S1. AP model was stimulated by the application of pulses of a $1.25 \mathrm{~Hz}$ frequency, which roughly corresponds to $75 \mathrm{bpm}$ of normal human heart. Equation 3 was integrated by using the forward Euler method with a time step of $0.02 \mathrm{~ms}$. The Hodgkin-Huxley $(\mathrm{HH})$ equations for the gating variables of the various time-dependent currents including the modified $I_{\mathrm{K} 1}$ in the ten Tusscher et al. model were integrated using the Rush and Larsen scheme [34].

\section{Tissue simulations}

Initiation and conduction of APs in the multicellular tissue model were modeled with the following equation:

$$
\frac{\partial V}{\partial t}=-\frac{I_{\text {ion }}+I_{\text {stim }}}{C_{m}}+\nabla \cdot(D \nabla V)
$$

where $D$ is the diffusion coefficient between ventricular cells describing the tissue's electrical conductivity, and $\nabla$ is the gradient operator.

For one-dimensional (1D) simulations, a single fiber model of transmural strand with a length of $15 \mathrm{~mm}$ was used (discretized into 100 nodes). The total length is close to the normal range of the human transmural ventricle wall thickness of $\sim 4.0$ to $14.0 \mathrm{~mm}$ [35]. The fiber employed a spatial resolution of $0.15 \mathrm{~mm}$, with each node representing a $150-\mu \mathrm{M}$ cylindrical cell. The fiber model was composed of three regions containing: 
ENDO, MIDDLE and EPI cells (with respective proportions of 25\%: 35\%: 40\%). The lengths of each region were 3.75, 5.25 and $6 \mathrm{~mm}$ for ENDO, MIDDLE and EPI, respectively. These proportions and the total length are similar to those used in other studies [10, 36-38]. The diffusion coefficient, $D$ was set at $0.0008 \mathrm{~cm}^{2} / \mathrm{ms}$ giving a conduction velocity $(\mathrm{CV})$ of $52 \mathrm{~cm} / \mathrm{s}$ through the fiber, which is relatively close to the experimental $\mathrm{CV}$ of $\sim 50 \mathrm{~cm} / \mathrm{s}[39,40]$. In the model, a fivefold decrease in gap junctional conductance between the EPI and MIDDLE region was implemented. This followed the study of Gima and Rudy [41], which was based on experimental work of Yan et al. [35] showing a sharp transition in the tissue resistance in a left ventricular wedge, implying reduced electrical coupling in this region [42].

The pseudo-ECG was computed as an integral of the spatial gradient of transmembrane potential of cells at all positions on the fiber from an electrode by using [41]:

$$
\phi_{e}\left(x^{\prime}\right)=\frac{\alpha^{2}}{4} \int(-\nabla V) \cdot\left[\frac{1}{r}\right] d x
$$

where $\emptyset_{e}$ is the computed potential at the electrode, $\alpha$ is the radius of the strand, $r$ is the Euclidean distance from a source point $x$ to the electrode point $x^{\prime}$, and $d x$ is the spatial resolution. In this study, we placed the electrode at a position $2.0 \mathrm{~cm}$ from the ENDO end of the strand, which resulted in a negative QRS and a positive $\mathrm{T}$ wave in the pseudoECG waveforms. These waveforms were in concordance with the clinical data [2]. The QT interval was computed as the time interval determined by the definition of the onset of the QRS complex $\left(Q_{\text {onset }}=0.0 \mathrm{mV}, t=0 \mathrm{~ms}\right)$ and the point at which the end of the T-wave $\left(T_{\text {end }}\right)$ crossed the baseline. The time at which the ECG signal fell below a threshold $\left(V_{\text {thresh }}=0.01 \mathrm{mV}\right)$ was defined as $T_{\text {end }}$.

For two-dimensional (2D) simulations, an idealized geometry was implemented. The idealized geometry was a simple sheet of tissue measured $15 \mathrm{~mm}$ by $75 \mathrm{~mm}$. It was modeled by expanding the $1 \mathrm{D}$ fiber (100 cells, $15 \mathrm{~mm}$ in the $x$-direction) into a sheet with a width of $75 \mathrm{~mm}$ in the $y$-direction. The spatial resolution in both $x$ and $y$ directions was the same as used in the $1 \mathrm{D}$ fiber. In the $2 \mathrm{D}$ sheet tissue, re-entry was initiated by an S1-S2 stimulation protocol. A planar wave was initiated at the ENDO end by an S1 stimulus (amplitude: $-52 \mathrm{pA} / \mathrm{pF}$, duration: $1 \mathrm{~ms}$ ). During the vulnerable window, an S2 stimulus with the same amplitude and duration as the $\mathrm{S} 1$ was applied to the junction of the EPI to MIDDLE cells. This action led to the initiation of a re-entrant excitation wave that then rotated through the tissue. However, the S2 had variable spatial sizes (width $0.45 \mathrm{~mm}$, length varies between 0 and $75 \mathrm{~mm}$ ). A S2 stimulus with sufficient spatial size (S2 size) in 2D tissue is required to provide an adequate re-entrant pathway, which is dependent on the wavelength (wavelength $=\mathrm{ERP} \times \mathrm{CV}$ ) of re-entry. In order to evaluate the critical size of the re-entrant pathway, we estimated the minimum length of S2 stimulus. The minimum length was measured as the smallest length which supported the formation of re-entry by decreasing it from 75 to $0 \mathrm{~mm}$ with decrement of $0.15 \mathrm{~mm}$. This minimum length gives an indication of the susceptibility of the tissue to sustain reentry, i.e., the larger the minimum length, the more difficult for the initiation of re-entry [37]. For such an initiated re-entrant excitation waves, effects of amiodarone on their dynamical behaviors were evaluated in the SQT3 condition. 


\section{Results}

\section{Effects of amiodarone on SQT3 in the cell model}

Figure 2 shows the results of simulated current-voltage $(I-V)$ for WT and mutant $I_{\mathrm{K} 1}$ channels. In simulations, $I_{\mathrm{K} 1}$ was elicited by $400 \mathrm{~ms}$ depolarizing voltage steps from -120 to $+20 \mathrm{mV}$ and from a holding potential of $-60 \mathrm{mV}$, with current traces for $\mathrm{WT}$, WT-D172N and D172N $I_{\mathrm{K} 1}$ current shown respectively in Fig. 2a-c. Figure 2d-f shows current-voltage $(I-V)$ data for WT, WT-D172N and D172N conditions. The reversal potential of the $I-V$ relations for WT and mutant (WT-D172N and D172N) $I_{\mathrm{K} 1}$ were not significantly different. However, outward $I_{\mathrm{K} 1}$ current differed markedly between WT and mutant channels: it was significantly greater for WT-D172N and D172N conditions than for WT condition. These features are consistent with those experimental data reported previously [2].

Changes in $I_{\mathrm{K} 1}$ due to the $K C N J 2 \mathrm{D} 172 \mathrm{~N}$ mutation abbreviated human ventricular $\mathrm{APD}$ as shown in Fig. 3. Figure $3 \mathrm{a}-\mathrm{c}$ shows AP waveforms $V(\mathrm{mV})$ versus time $t(\mathrm{~ms})$. The measured $\mathrm{APD}_{90}$ was 302, 406 and $304 \mathrm{~ms}$ for the ENDO, MIDDLE and EPI cell, respectively, in the WT condition, which were shortened, respectively, to 273, 357 and $274 \mathrm{~ms}$ for the WT-D172N condition and to 261, 341 and $262 \mathrm{~ms}$ for the D172N condition. The abbreviated APD resulted from an increased $I_{\mathrm{K} 1}$ during the AP repolarization phase as shown in Fig. $3 d-f$.

The effects of different doses of amiodarone on the AP characteristics for the human ventricular EPI cells are shown in Fig. 4a (WT-D172N condition) and b (D172N condition), and the corresponding $\mathrm{APD}_{90}$ are plotted in Fig. $4 \mathrm{c}$ and $\mathrm{d}$. The measured $\mathrm{APD}_{90}$ was prolonged from $275 \mathrm{~ms}$ in WT-D172N to 291 and 289 in the presence of low and high doses of amiodarone, respectively, and from $263 \mathrm{~ms}$ in D172N condition to 277 and $273 \mathrm{~ms}$ in the presence of low and high doses of amiodarone. Additional simulations on ENDO and MIDDLE cells (data not shown) showed similar effects of amiodarone to those seen with the EPI ventricular AP waveform.

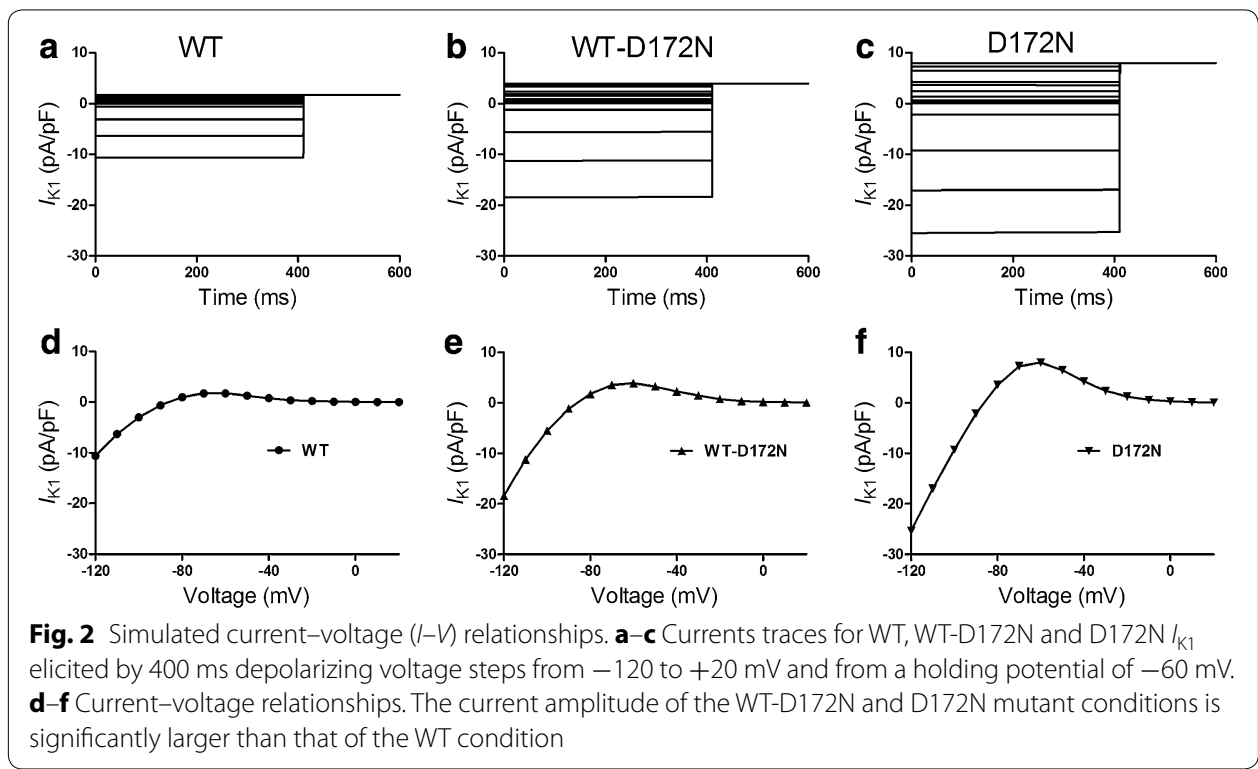



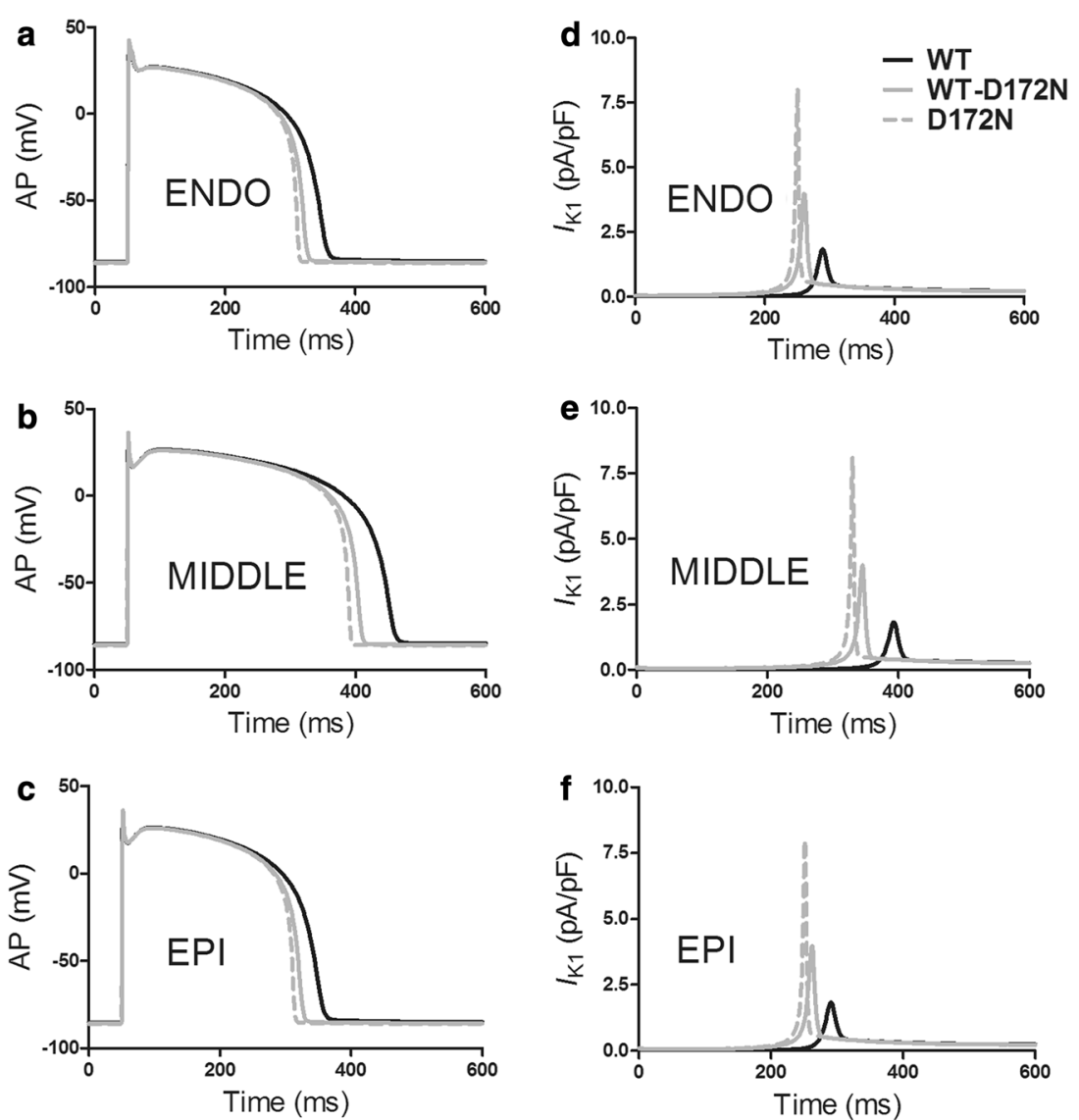

Fig. 3 Simulations of APs together with the corresponding time course and amplitude of $I_{K 1}$ in the WT, WTD172N and D172N conditions. a, d APs and $I_{\mathrm{K} 1}$ for ENDO cells. b, e APs and $I_{\mathrm{K} 1}$ for MIDDLE cells. c, f APs and $I_{\mathrm{K} 1}$ for EPI cells

Rate-dependent effects of amiodarone on the APD of EPI cells was shown in Fig. 5a and $\mathrm{b}$ for the computed APD restitution (APD-R) curves for WT and mutation conditions. Across the range of diastolic intervals (DI) studied, the measured $\mathrm{APD}_{90}$ was increased in the presence of amiodarone. In addition, the APD-R relationships were attenuated by the action of amiodarone, as indicated by the decreased maximal slope of the APD-R curves (Fig. 5c, d). The measured ERP was also increased in the presence of amiodarone. The ERP reduction was also rate-dependent in Fig. 5e and f. Across a range of BCLs, the measured ERP in the presence of amiodarone was increased. Actions of amiodarone also attenuated the ERP-R curves, as indicated by the decreased maximal slope of the ERP-R curve (Fig. $5 \mathrm{~g}, \mathrm{~h}$ ) in the presence of amiodarone. As a decreased steepness of APD-R and ERP-R curves are believed to be associated with stability of reentry [43], indicating anti-arrhythmic effects of amiodarone on SQT3.

\section{Effects of amiodarone on SQT3 in the 1D fiber model}

Using a 1D-fiber model, a pseudo-ECG was computed for the WT, WT-D172N, D172N and actions of amiodarone conditions (Fig. 6). The results are shown in Fig. 6a, b, e and $\mathrm{f}$ for an excitation wave propagating from ENDO towards MIDDLE and EPI parts of the fiber. Amiodarone delayed the repolarization phase both in the WT-D172N and 

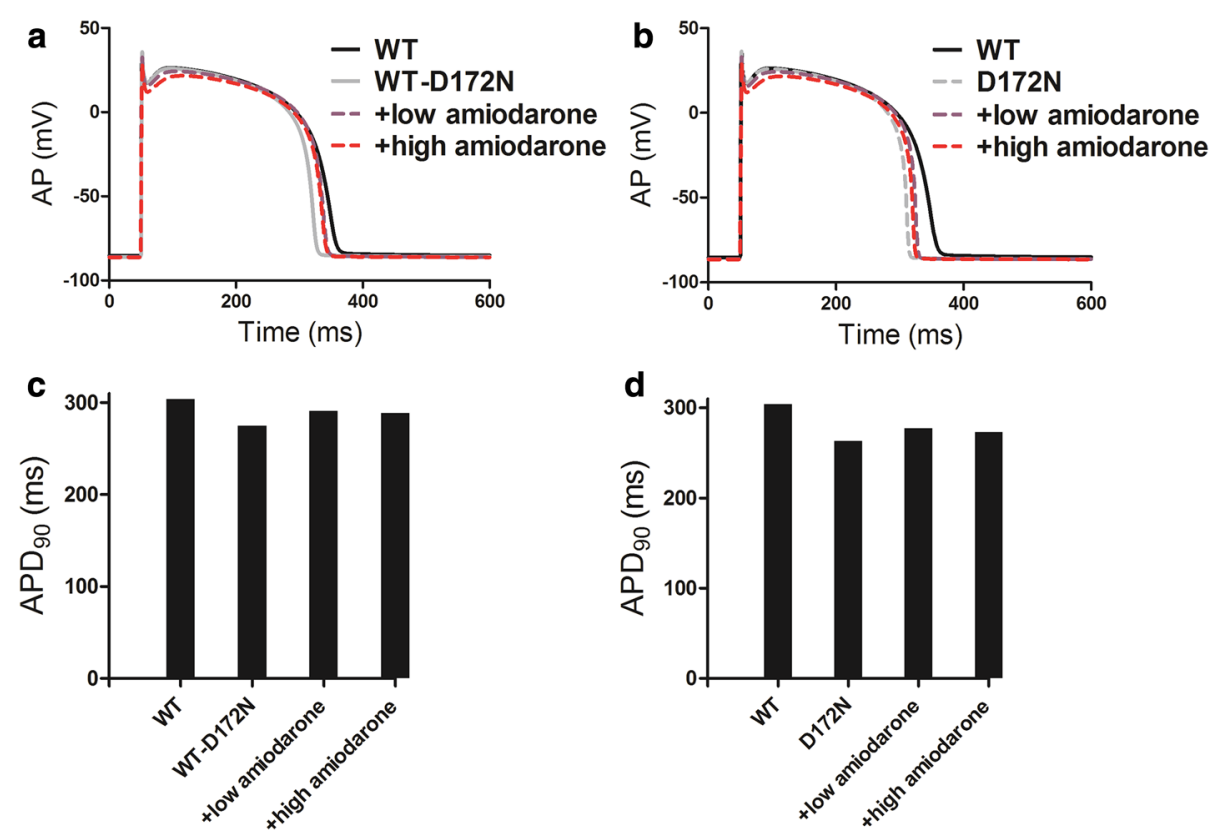

Fig. 4 Effects of low and high doses of amiodarone on human ventricular EPI cell. a, c APs under the WTD172N condition in the presence of amiodarone, and corresponding APD 90 histogram. $\mathbf{b}, \mathbf{d}$ APs under the D172N condition in the presence of amiodarone, and corresponding APD 90 histogram

D172N conditions. These changes of the excitation wave were also reflected in the computed pseudo-ECGs as shown in Fig. 6c, d, g and h for the WT-D172N, D172N and amiodarone-in-action conditions respectively. To quantify the effects, we calculated the QT intervals extracted from the simulation data (Fig. 6i-l). In simulations, the mutation shortened APD, leading to abbreviated QT interval (322 ms for WT-D172N condition), which is similar to clinical observation (315 ms for the proband and $320 \mathrm{~ms}$ for her father) [2]. The simulated mutation produced a taller T-wave amplitude, which is also similar to clinical observation [2]. The pseudo-ECGs show an increment in the QT interval in the presence of low and high doses of amiodarone, which changed from $322 \mathrm{~ms}$ in the WT-D172N condition to 341 and $340 \mathrm{~ms}$, respectively. For the same condition, the QT interval was prolonged from $308 \mathrm{~ms}$ in the D172N condition to 325 and $324 \mathrm{~ms}$, respectively. These QT intervals were prolonged, but were not within the normal physiological range (from 360 to $440 \mathrm{~ms}$ ). The simulated small QT interval prolongation was consistent with the clinical study [18] in which the patient remained short QT after use of amiodarone. T-wave amplitude in the presence of amiodarone under WT-D172N and D172N conditions was decreased as shown in Fig. 6i and k.

An increase in T-wave amplitude during simulated SQTS has been attributed to an increased spatial gradient in membrane potential $[36,37]$ and an inverse effect might explain the decrease T-wave amplitude seen in Fig. 6 of this study. Therefore, we examined effects of amiodarone on the membrane potential heterogeneity $(\delta V)$ which was calculated as the difference between APs among three cell types. Via cell-to-cell electrical interaction, $\delta V$ (in the temporal domain) is smoothed in space producing a spatial gradient $\nabla V$ (in the spatial domain), which contributes to the computed pseudo-ECG as described in Eq. 4. Figure 7a and b show simulated ENDO, MIDDLE and EPI APs with 

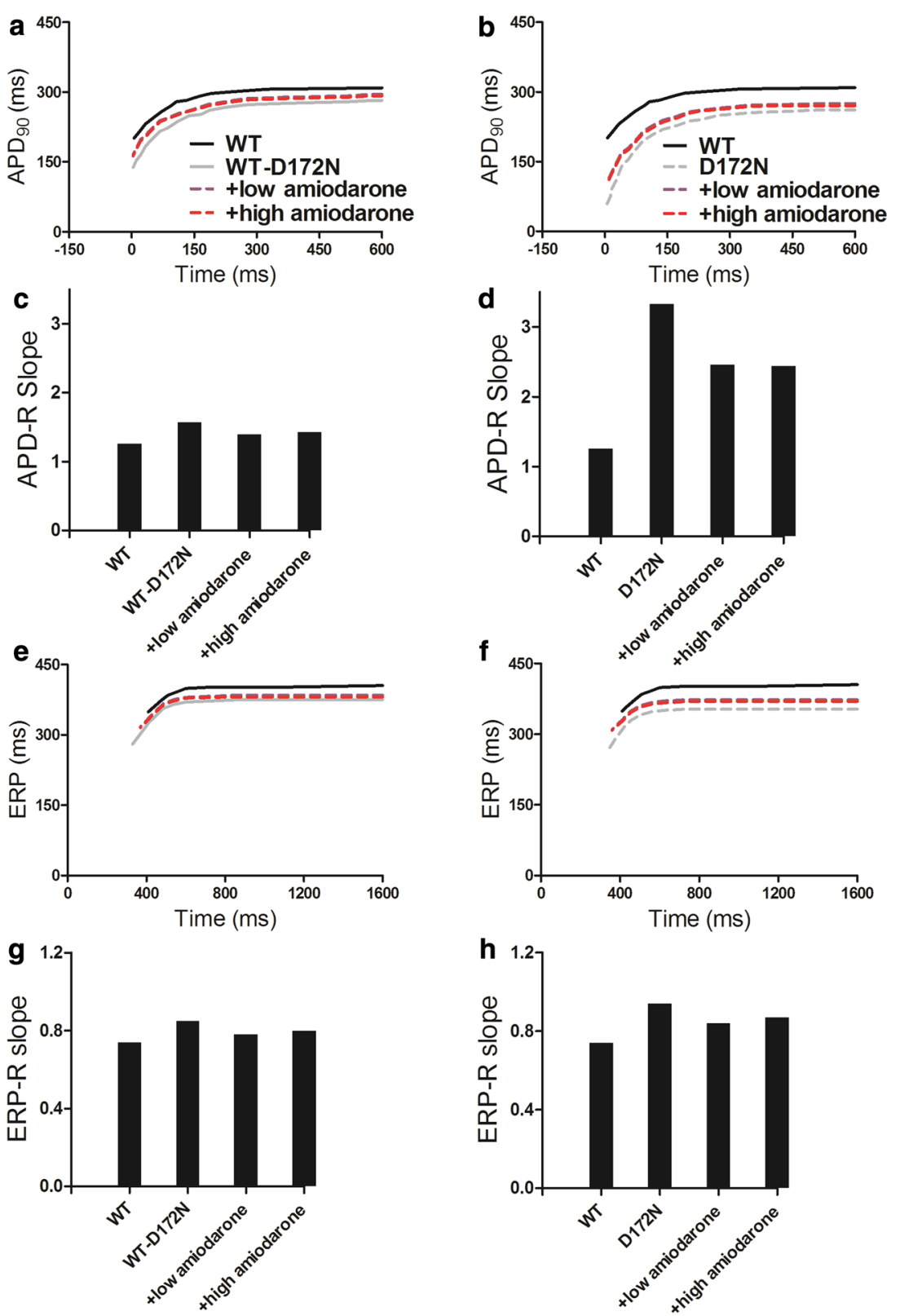

Fig. 5 Rate-dependent APD restitution curves and ERP restitution curves for EPI cells under the WT, WTD172N and D172N conditions, and in the presence of amiodarone. $\mathbf{a}, \mathbf{b}$ APD restitution curves under the WT-D172N and D172N conditions, respectively. c, d Measured slopes of APD restitution curves under the WT-D172N and D172N conditions, respectively. e, f ERP restitution curves under the WT-D172N and D172N conditions, respectively. $\mathbf{g}$, h Measured slopes of ERP restitution curves under the WT-D172N and D172N conditions, respectively

WT-D172N and WT-D172N + low amiodarone conditions whilst Fig. 7c and d show corresponding time-course plots of the $\delta V$ during APs between ENDO, MIDDLE and EPI cells. The maximal $\delta V$ between both ENDO-MIDDLE and MIDDLE-EPI APs was smaller with the use of amiodarone than before (Fig. 7e, f). The actions of amiodarone also augmented $\mathrm{APD}_{90}$ dispersion in the fiber model with the electrotonic interaction 


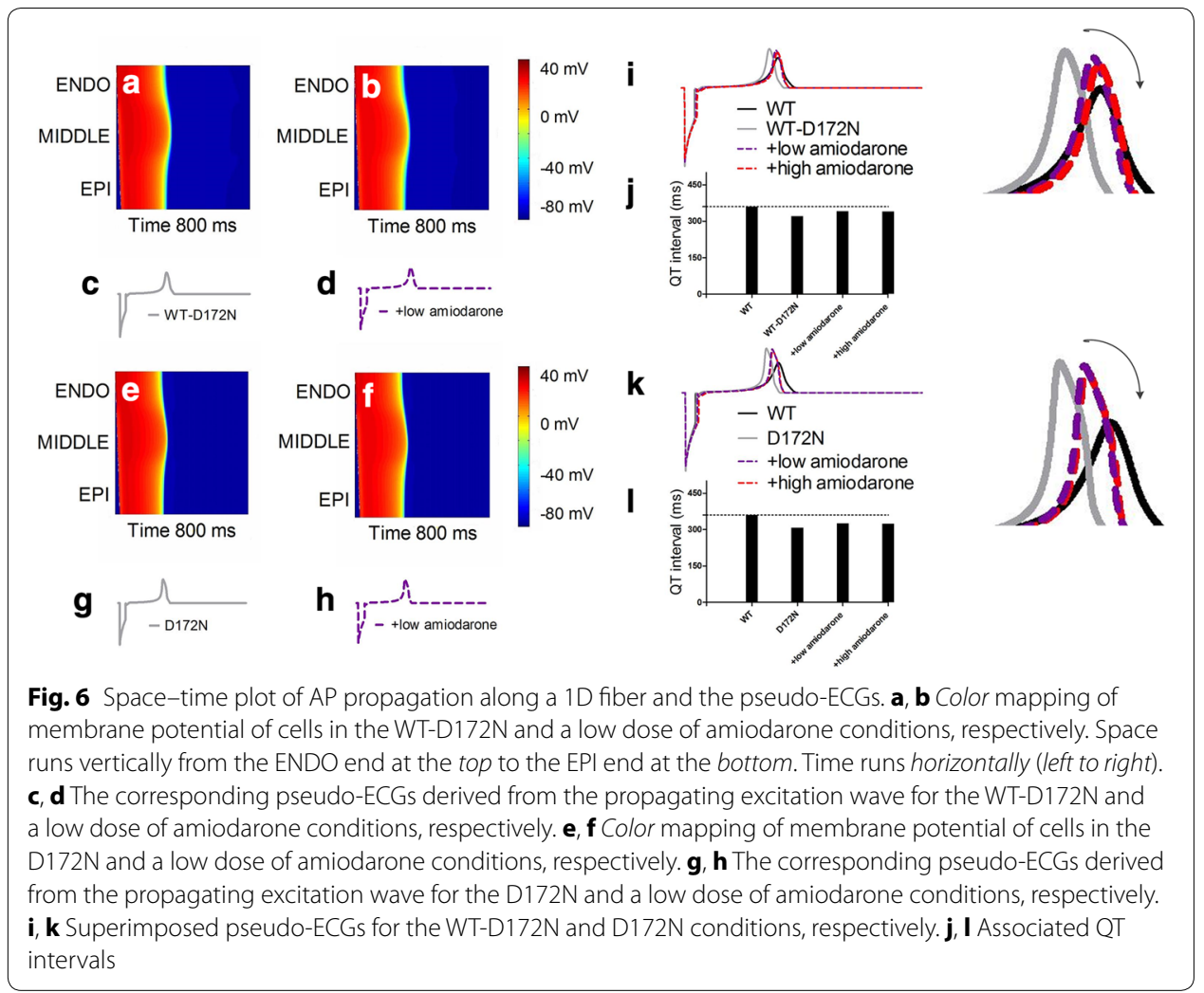

between cells. Figure $7 \mathrm{~g}$ shows the spatial distribution of $\mathrm{APD}_{90}$ for WT, WT-D172N and amiodarone-in-action conditions. The absolute spatial gradient of $\mathrm{APD}_{90}$ was found to be augmented, as shown in Fig. 7h. The maximal gradient across the 1D fiber was smaller with the use of amiodarone than before (Fig. 7i, j). The D172N mutation augmented the APD dispersion at the junction region between the MIDDLE and EPI regions, whilst amiodarone attenuated the APD dispersion across the 1D strand, which also led to the decreased T-wave amplitude.

Simulations were also performed to determine whether or not amiodarone decreased susceptibility of tissue to ventricular arrhythmogenesis. Using a 1D fiber model we quantified tissue's temporal vulnerability to unidirectional conduction in response to a premature stimulus for the amiodarone-in-action condition. Figure $8 \mathrm{a}-\mathrm{c}$ shows the conditioning excitation wave and the response of the tissue to a premature stimulus applied at ENDO part of the fiber (marked by the arrow). In Fig. 8a, the premature stimulus was applied early (i.e., at time $=335 \mathrm{~ms}$ after the previous excitation wave) so that the tissue surrounding the stimulus site did not have enough time to recover for re-excitation to occur. As a consequence, a bidirectional block was observed. In Fig. 8b, the premature stimulus was applied within the vulnerable window (at time $=341.5 \mathrm{~ms}$ ) that produced a unidirectional conduction block. In Fig. 8c, the premature stimulus was applied after the vulnerable window (at time $=350 \mathrm{~ms}$ ) and, consequently, bidirectional conduction was observed. Figure $8 \mathrm{~g}$ and $\mathrm{h}$ show the width of the vulnerable window during which a premature stimulus applied at the ENDO part of the fiber resulted in the unidirectional block under WT, WT-D172N, D172N and amiodarone-in-action conditions. 


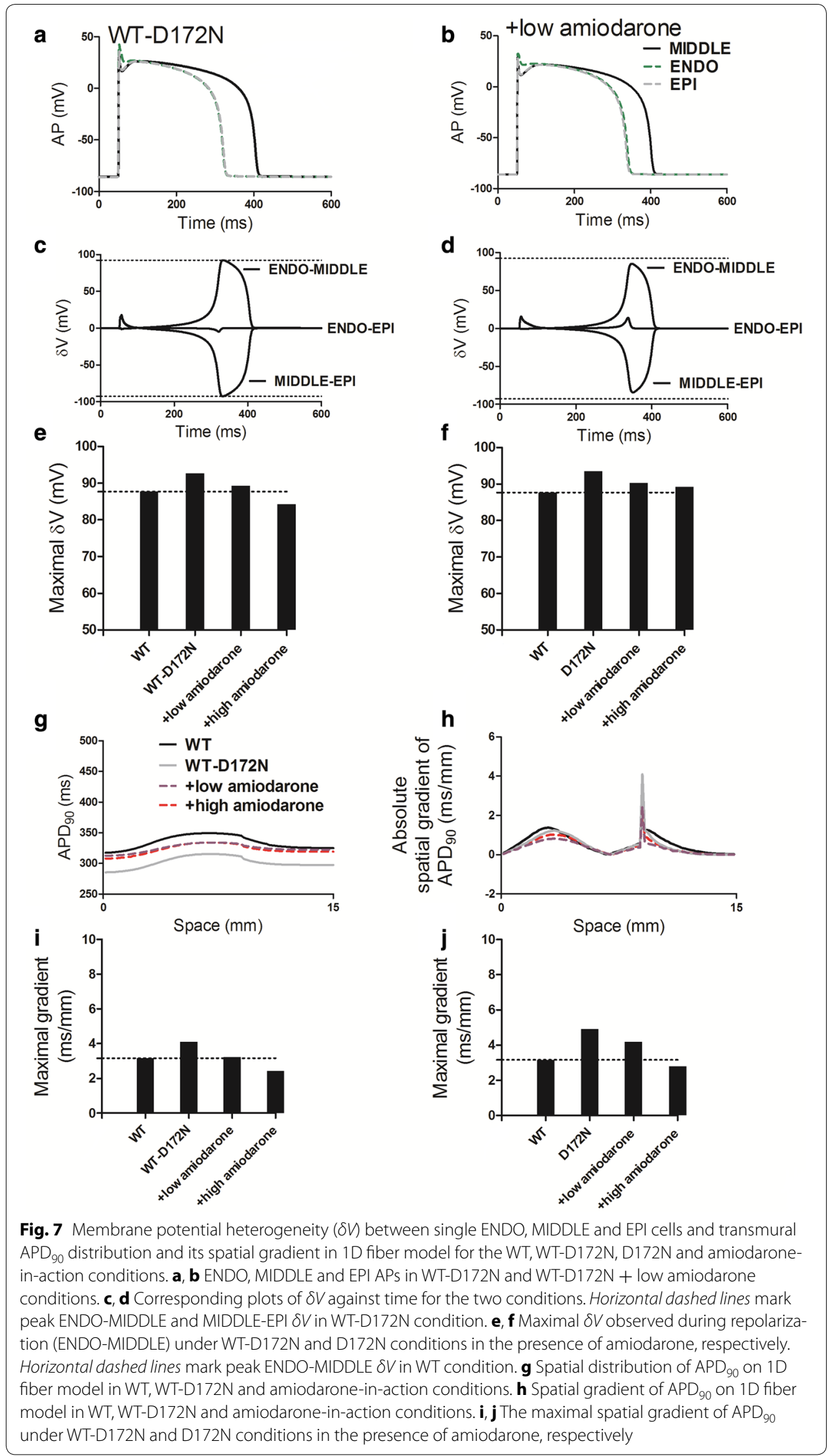




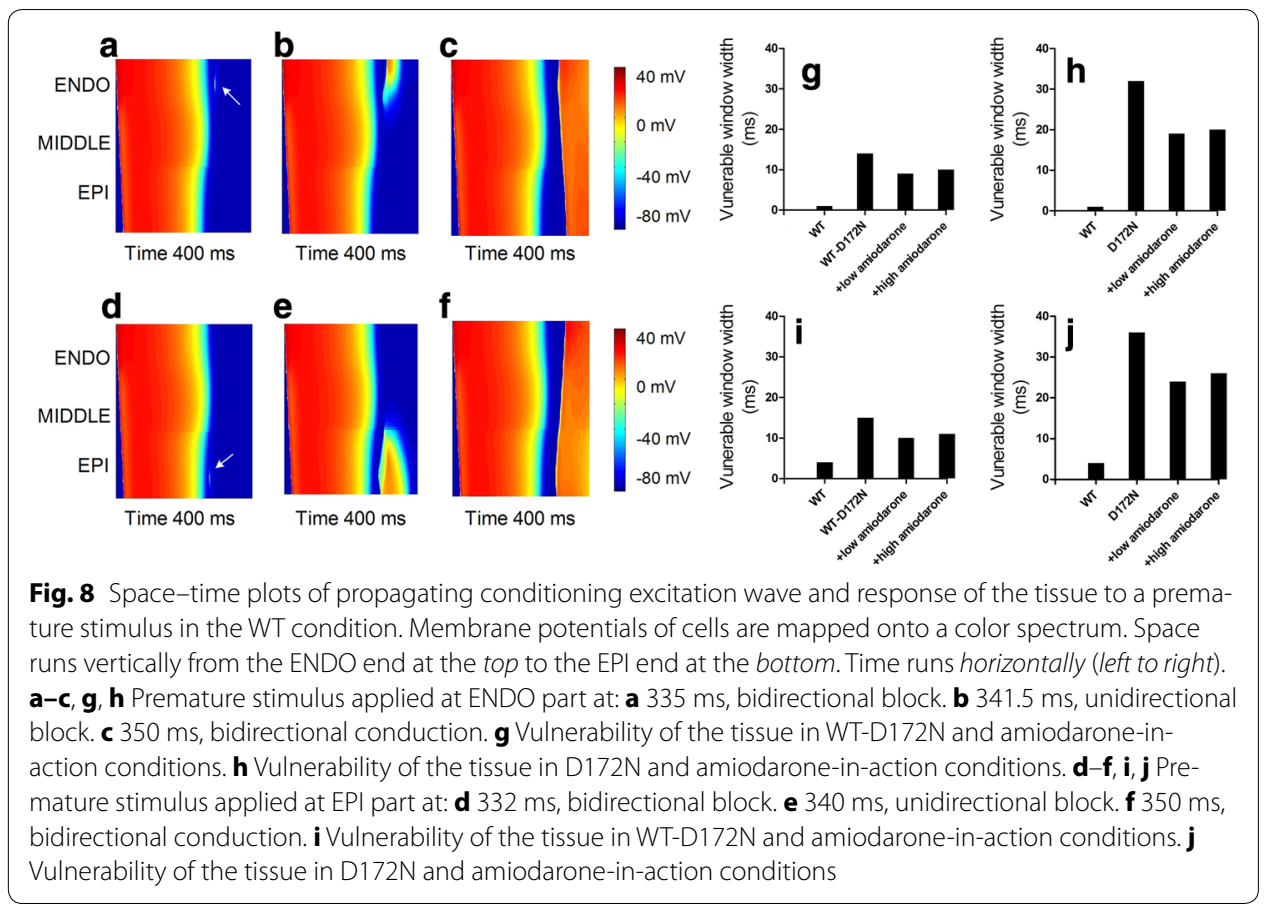

Simulations were also performed in which the premature stimulus was applied to the EPI rather than the ENDO part of the fiber (Fig. $8 \mathrm{~d}-\mathrm{f}$ ). Figure $8 \mathrm{~d}-\mathrm{f}$ represent bidirectional conduction block (at time $=332 \mathrm{~ms}$ ), unidirectional block (at time $=340 \mathrm{~ms}$ ) and bidirectional conduction (at time $=350 \mathrm{~ms}$ ). Effects of amiodarone on temporal vulnerability in WT-D172N and D172N mutations are shown in Fig. $8 \mathrm{i}$ and $\mathrm{j}$. These simulation results show clearly that the tissue's temporal vulnerability is decreased by amiodarone.

\section{Effects of amiodarone on SQT3 in the 2D tissue model}

Using an idealized 2D sheet model, we proceeded to measure the minimum spatial size of a premature S2 stimulus that provides a sufficient substrate size for sustaining re-entry initiated for SQT3 and amiodarone-in-action conditions. Results are shown in Fig. 9, in which patterns of re-entrant excitation waves are represented via pseudo-color mapping of transmembrane voltage. During the simulation, the S1 stimulus was applied to the ENDO end to evoke a planer excitation wave that propagated towards the MIDDLE and EPI regions (Fig. 9a, f, k). After a time delay (WT: 340 ms; WT-D172N: 312 ms; WT-D172N + low amiodarone: $325 \mathrm{~ms}$ ), a S2 stimulus was applied to the MIDDLE-EPI junction region that produced a unidirectional conduction, forming a re-entrant excitation wave (Fig. 9b, g, l). The induced re-entrant excitation self-terminated for the WT condition (Fig. 9c-e), but sustained for the WT-D172N mutation condition (Fig. 9h-j). It is attributable to the shortening of APD due to the mutation, decreasing the wavelength of the ventricular excitation wave. With the use of amiodarone, re-entrant excitation wave self-terminated as shown in Fig. 9m-o. Figure 9p-r show a recording of the evolution of the AP of an EPI cell in the 2D sheet model under the WT, WT-D172N and low dose of amiodarone conditions, respectively. The measured minimum spatial length of S2 stimulus required for re-entry under WT-D172N and D172N conditions in 


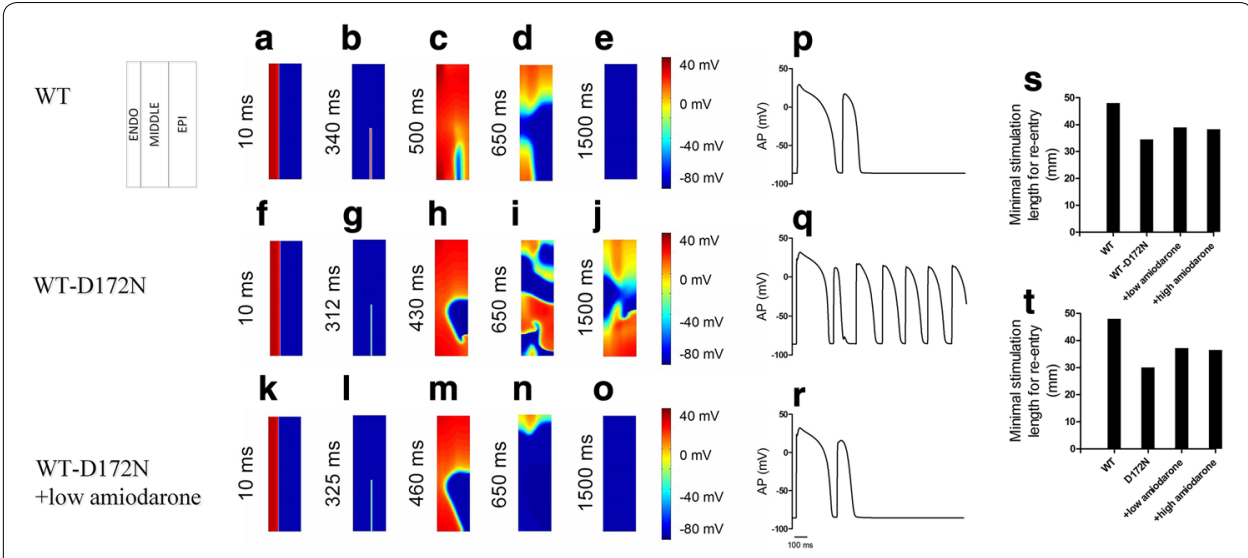

Fig. 9 Snapshots of initiation and conduction of re-entrant excitation wave in a 2D model of transmural ventricle tissue under the WT, WT-D172N and amiodarone-in-action conditions. a, f, k A planar wave generated by S1 stimulus at the ENDO end, which propagates towards the MIDDLE and EPI regions. Snapshots at time $=10 \mathrm{~ms}$. b, $\mathbf{g}$, I S2 was applied to the MIDDLE-EPI junction region during the vulnerable window of the tissue. $\mathbf{c}, \mathbf{h}, \mathbf{m}$ Developed re-entrant excitation waves. $\mathbf{d}, \mathbf{i}, \mathbf{n}$ Developed re-entrant excitation waves. Snapshots at time $650 \mathrm{~ms}$. e, j, o Snapshots of the re-entrant excitation waves. Re-entry was prevented in the presence of amiodarone. Snapshots at time $1500 \mathrm{~ms}$. p, q, r Evolution of the AP of an EPI cell under the WT, WT-D172N and WT-D172N + low amiodarone conditions, respectively. s, $\mathbf{t}$ The measured minimum spatial length of $\mathrm{S} 2$ that provides a sufficient substrate for the formation of a re-entrant circuit under WT-D172N and D172N conditions in the presence of amiodarone, respectively

the presence of amiodarone is shown in Fig. 9s and t, respectively. Prolongation of APD due to the actions of amiodarone increased the wavelength of the ventricular excitation wave, and thus increased the minimal length of S2 stimulus. This result supports the notion that in SQT3 tissue with the use of amiodarone, re-entrant excitation waves is less likely to occur.

\section{Discussion}

\section{Summary of the major findings}

The proband in whom the KCNJ2 D172N mutation was identified was heterozygotic for the SQT3 mutation (WT-D172N) [2]. She had a QTc interval of $315 \mathrm{~ms}$, whereas her father had a QTc interval of $320 \mathrm{~ms}$ [2]. It is of particular note that with the use of amiodarone in the WT-D172N condition, mimicking the effects on the heterozygous state of the proband, we found that the simulated QT interval extended from 322 to $341 \mathrm{~ms}$ in the low-dose amiodarone condition and $340 \mathrm{~ms}$ in the high-dose condition. The QT interval extended from $308 \mathrm{~ms}$ in the D172N condition to $325 \mathrm{~ms}$ in the low-dose amiodarone condition and $324 \mathrm{~ms}$ in the high-dose condition. In addition, the risk of arrhythmogenesis as measured by the vulnerability of tissue was decreased in the presence of both low and high doses of amiodarone. Our simulation results indicate that the amiodarone (i) extended the $\mathrm{APD}_{90}$ in the SQT3 condition and decreased susceptibility to arrhythmia; (ii) extended the QT interval on the pseudo-ECG and reduced the T-wave amplitude; (iii) decreased the maximal voltage heterogeneity $(\delta V)$ during APs, which contributed to the decreased T-wave amplitude; (iv) prolonged cell $\mathrm{APD}_{90}$ across the $1 \mathrm{D}$ strand and decreased maximal dispersion of $\mathrm{APD}_{90}$, which also subsequently led to the decreased T-wave amplitude; (v) prevented re-entrant excitation waves and increased the minimum substrate size of tissue required to maintain re-entrant excitation waves. 
These changes due to the presence of amiodarone indicated its anti-arrhythmic effects in SQT3 condition.

\section{Relevance of the study}

SQT3 produces changes on the QT interval and T-wave, caused by a shortening of total repolarization time, which are in agreement with the previous studies $[2,10]$. Those studies investigated the $I_{\mathrm{K} 1}$ current underlying the changes in the pseudo-ECG caused by a D172N mutation in the SQT3 condition. However, the present study first assessed the pharmacological effect of amiodarone on ventricular excitation associated with SQT3. Our simulations were able to reproduce the known effects of D172N mutation on the electrophysiological activity of the ventricles as shown in the literature [2], which provide the first step towards the validation of the use of the cardiac models in assessing the effects of amiodarone on SQT3.

\section{Significance of the study}

Our simulations showed the anti-arrhythmic effects of amiodarone on SQT3. This was done by thoroughly analyzing the functional effects of amiodarone at the cell and tissue levels. First, the action of amiodarone was simulated by using a single pore block theory, as in the previous studies $[44,45]$. Then, the effects of amiodarone were investigated in a $1 \mathrm{D}$ ventricular model, using which pseudo-ECGs were simulated. Finally, effects of amiodarone on re-entrant excitation wave dynamics in SQT3 condition were quantitatively assessed. Our results showed that both of low and high doses of amiodarone exhibited anti-arrhythmic effects for SQT3.

The results demonstrated that the presence of amiodarone led to QT interval prolongation and a reduction in the T-wave amplitude. As expected, the QT interval prolongation is explained by an APD prolongation, whereas the decrease in the T-wave amplitude correlates with reduced dispersion in repolarization as demonstrated by decreased dispersion of the $\mathrm{APD}_{90}$ across the 1D fiber model.

In the SQT3 setting, ventricular fibrillation could be elicited by programmed electrical stimulation. It was hypothesized that APD and ERP abbreviation are factors contributing to an increased risk of re-entrant arrhythmias. Amiodarone prevented re-entrant excitation waves. This is attributable to the APD prolongation, which increased the wavelength of excitation waves, and therefore, increased the critical mass of tissue necessary to accommodate re-entrant excitation waves, suppressing the maintenance of reentrant excitation waves. Thus, re-entrant excitation wave in the presence of amiodarone becomes more difficult to be initiated and sustained. This simulation result was consistent with no further syncope or palpitations in clinical study [18].

In this study, due to the scarcity of accurate experimental models of SQT3 and of in vitro pharmacological data of SQT3, we are currently unable to compare our simulation results with experiments for validation. However, we used the well-established ten Tusscher et al. [21] model for simulating human ventricular APs along with the recent SQT3 $I_{\mathrm{K} 1}$ model developed by our group [10]. These models were validated previously. With regard to amiodarone effects, although there are no direct experimental data for us to compare, results from the clinical study [18] have demonstrated the beneficial effects of amiodarone on SQTS patients. Our simulation data are in accordance with the 
clinical phenomenon [18], indicating the anti-arrhythmic effect of amiodarone in SQT3 condition.

\section{Limitations of the study}

Limitations of the ten Tusscher et al. [21] model have been discussed in detail elsewhere. Although such a model is not as complete as the newest models [33], it is sufficient for the purpose of this study as the simulated effect of amiodarone on SQT3 is in accordance with the clinical phenomenon [18]. The biophysical approach, which we adopt here, has also been used by several other groups (see, e.g. [38, 46, 47]).

Although we considered intercellular electrical coupling with each distinct cell type, the 1D and 2D idealized models cannot represent a realistic anatomical structure, which might influence the maintenance of ventricular arrhythmias. The structure of realistic anatomical tissue is much more complex than the idealized isotropic tissue studied here [48]. The idealized model, which was used to simulate re-entrant excitation waves, lacks inclusion of Purkinje fibers, which may play a significant role in arrhythmogenesis in SQTS. Furthermore, the model did not consider the effect of the atria, mechano-electric coupling and feedback, tissue deformation and coronary flow, which feasibly might influence re-entrant excitation waves.

Regarding the experimental conditions, many factors that may affect the binding of a drug to ion channels are increasingly being modeled, including oxygen, the concentration of ions, temperature and $\mathrm{pH}$ [49]. However, pharmaceutical screening does not usually record sufficient data to evaluate the dynamic changes caused by drugs [45]. For conditions with variable pacing rates, conductance block approximation may be insufficient for simulation of realistic amiodarone actions.

Another limitation is the use of data from animal models instead of human to mathematically describe the effects of amiodarone on the ionic currents as listed in Table 1. Limitations of data from animal models of disease in nonclinical safety testing include a lack of historical control, heterogeneity in disease expression, a limited life span and confounding effects of the disease. However, little information is known about the effects of amiodarone on the specific ionic currents in human. Therefore, for future work, integration of experimental data for the different ion channels of human heart acquired at body temperature when become available could provide a better insight into the effects of amiodarone on SQT3.

\section{Conclusions}

In this study, we presented a simulation study showing the effects of amiodarone on SQT3. It was shown that amiodarone altered the profiles of APs and pseudo-ECGs, as well as the dynamics of re-entrant excitation waves. Amiodarone produced QT interval prolongation (owing to APD prolongation), decreased APD dispersion and membrane potential dispersion $(\delta V)$, which contributed to the decreased T-wave amplitude. Additionally, amiodarone increased ERP, reduced vulnerable window and prevented reentrant excitation waves. Our results demonstrate anti-arrhythmic effects of amiodarone on SQT3 and suggest that amiodarone may be a potential pharmacological agent for treating SQT3 patients. 


\section{Additional file}

Additional file 1. An appendix showing $I_{K 1}$ model equation parameters for WT, WT-D172N and K1 D172N conditions.

\section{Abbreviations}

SQTS: short QT syndrome; SCD: sudden cardiac death; $\left.\right|_{k 1}$ : inward rectifier potassium channel current; APD: action potential duration; ERP: effective refractory period; APs: action potentials; $1 \mathrm{D}$ : one-dimensional; $2 \mathrm{D}$ : two-dimensional; $\delta \mathrm{V}$ : maximal voltage heterogeneity; SQT3: KCNJ2-lined short QT syndrome; QTc: corrected QT interval; ECG: electrocardiogram; APD-R: APD restitution; ERP-R: ERP restitution; ICD: implantable cardioverter defibrillator; ENDO: endocardial; MIDDLE: mid-myocardial EPI: epicardial; WT: wild-type; $I_{50}$ : half maximal inhibitory concentration; $\mathrm{nH}$ : Hill coefficient; $I_{\mathrm{Na}}$ : late sodium current; BCL: basic cycle length; HH: Hodgkin-Huxley; CV: conduction velocity; I-V: current-voltage; Dl: diastolic interval.

\section{Authors' contributions}

$\mathrm{KW}$ and $\mathrm{HZ}$ conceived and designed the experiments. $\mathrm{CL}$ performed the experiments and analyzed the results. $\mathrm{CL}$ wrote the manuscript. All authors read and approved the final manuscript.

\section{Authors' information}

CL was born in Jiangsu Province, China, in 1984. He is a Ph.D. student in the School of Computer Science of Technology, Harbin Institute of Technology, Harbin, China. He is also a joint Ph.D. student in the School of Physics and Astronomy, the University of Manchester, Manchester, United Kingdom, supported by the China Scholarship Council (CSC). His research focuses on computing in cardiology, and anti-arrhythmic drugs modeling and simulation.

KW was born in Sichuan Province, China, in 1964. He is Professor at Harbin Institute of Technology, China. He received his Ph.D. degree in computer science from Chongqing University, Chongqing, China, in 2001. Since 1998, he has been working in the School of Computer Science and Technology, Harbin Institute of Technology, China. Meanwhile, from 2000 to 2003 has been a Visiting Scholar at the Hong Kong Polytechnic University supported by Hong Kong Croucher Funding and from 2003 to 2004 he was a Research Fellow in the same university. Currently, he is a Professor and a supervisor of Ph.D. degree candidates in the School of Computer Science and Technology, Harbin Institute of Technology. His research interests include biometrics, image processing, pattern recognition, and biomedical engineering.

HZ was born in Anhui Province, China, in 1964. He is Professor and Chair of Biological Physics at the University of Manchester, United Kingdom. He received his Ph.D. degree in Computational Physiology from the University of Leeds in 1994. Then he worked as postdoctoral research fellow at Johns Hopkins University School of Medicine (1994-1995) and the University of Leeds (1996-2000), and then a senior research fellow at the University of Leeds (2000-2001). In October 2001, he moved to UMIST to take up a lectureship. From then, he worked as lecturer (2001-2004; UMIST), senior lecturer (2004-2006) and Reader (2006-2009) at the University of Manchester. In 2009, he was promoted to Chair, Professor of Biological Physics. In 2012, he was appointed as a Chinese National 1000-Plan Scholar. Currently, he holds a position of Distinguished Professor in Harbin Institute of Technology. His current research interests cover Predictive and Integrative Biology, pioneering the development of a virtual heart-a large-scale computer model of physiologically detailed heart.

\section{Author details}

${ }^{1}$ School of Computer Science and Technology, Harbin Institute of Technology (HIT), Harbin 150001, China. ${ }^{2}$ School of Physics and Astronomy, The University of Manchester, Manchester M13 9PL, UK. ${ }^{3}$ Space Institute of Southern China, Shenzhen 518117, China.

\section{Acknowledgements}

The authors would like to thank Dr. Ismail Adeniran for useful discussion. The authors are also grateful to the anonymous reviewers for their valuable comments and suggestions.

\section{Competing interests}

The authors declare that they have no competing interests.

Availability of data and materials

The Additional file for this article can be found online.

\section{Funding}

Financial support was provided by the China Scholarship Council (CSC) (to CL), and National Natural Science Foundation of China (NSFC) under Grant Nos. 61571165 and 61572152 (to KW and HZ).

\section{Publisher's Note}

Springer Nature remains neutral with regard to jurisdictional claims in published maps and institutional affiliations.

Received: 23 March 2017 Accepted: 1 June 2017

Published online: 07 June 2017 


\section{References}

1. Hong K, Bjerregaard P, Gussak I, Brugada R. Short QT syndrome and atrial fibrillation caused by mutation in KCNH2. J Cardiovasc Electrophysiol. 2005;16(4):394-6.

2. Priori SG, Pandit SV, Rivolta I, Berenfeld O, Ronchetti E, Dhamoon A, Napolitano C, Anumonwo J, di Barletta MR, Gudapakkam S, et al. A novel form of short QT syndrome (SQT3) is caused by a mutation in the KCNJ2 gene. Circ Res. 2005;96(7):800-7.

3. Bellocq C, van Ginneken AC, Bezzina CR, Alders M, Escande D, Mannens MM, Baro I, Wilde AA. Mutation in the KCNQ1 gene leading to the short QT-interval syndrome. Circulation. 2004;109(20):2394-7.

4. Brugada R, Hong K, Dumaine R, Cordeiro J, Gaita F, Borggrefe M, Menendez TM, Brugada J, Pollevick GD, Wolpert C, et al. Sudden death associated with short-QT syndrome linked to mutations in HERG. Circulation. 2004;109(1):30-5.

5. Gaita F, Giustetto C, Bianchi F, Wolpert C, Schimpf R, Riccardi R, Grossi S, Richiardi E, Borggrefe M. Short QT syndrome: a familial cause of sudden death. Circulation. 2003;108(8):965-70.

6. Gussak I, Brugada P, Brugada J, Wright RS, Kopecky SL, Chaitman BR, Bjerregaard P. Idiopathic short QT interval: a new clinical syndrome? Cardiology. 2000;94(2):99-102.

7. Antzelevitch C, Pollevick GD, Cordeiro JM, Casis O, Sanguinetti MC, Aizawa Y, Guerchicoff A, Pfeiffer R, Oliva A, Wollnik $B$, et al. Loss-of-function mutations in the cardiac calcium channel underlie a new clinical entity characterized by ST-segment elevation, short QT intervals, and sudden cardiac death. Circulation. 2007;115(4):442-9.

8. Templin C, Ghadri JR, Rougier JS, Baumer A, Kaplan V, Albesa M, Sticht H, Rauch A, Puleo C, Hu D, et al. Identification of a novel loss-of-function calcium channel gene mutation in short QT syndrome (SQTS6). Eur Heart J. 2011;32(9):1077-88.

9. Schimpf R, Wolpert C, Gaita F, Giustetto C, Borggrefe M. Short QT syndrome. Cardiovasc Res. 2005;67(3):357-66.

10. Adeniran I, El Harchi A, Hancox JC, Zhang H. Proarrhythmia in KCNJ2-linked short QT syndrome: insights from modelling. Cardiovasc Res. 2012;94(1):66-76.

11. Maury P, Extramiana F, Sbragia P, Giustetto C, Schimpf R, Duparc A, Wolpert C, Denjoy I, Delay M, Borggrefe M, Gaita F. Short QT syndrome. Update on a recent entity. Arch Cardiovasc Dis. 2008;101(11-12):779-86.

12. Schimpf R, Wolpert C, Bianchi F, Giustetto C, Gaita F, Bauersfeld U, Borggrefe M. Congenital short QT syndrome and implantable cardioverter defibrillator treatment: inherent risk for inappropriate shock delivery. J Cardiovasc Electrophysiol. 2003;14(12):1273-7.

13. Schimpf R, Bauersfeld U, Gaita F, Wolpert C. Short QT syndrome: successful prevention of sudden cardiac death in an adolescent by implantable cardioverter-defibrillator treatment for primary prophylaxis. Heart Rhythm. 2005;2(4):416-7.

14. Anttonen O, Junttila J, Giustetto C, Gaita F, Linna E, Karsikas M, Seppanen T, Perkiomaki JS, Makikallio TH, Brugada R, Huikuri HV. T-Wave morphology in short QT syndrome. Ann Noninvasive Electrocardiol. 2009;14(3):262-7.

15. Mondoly P, Cardin C, Rollin A, Duparc A, Maury P. Use of a subcutaneous ICD in a patient with short QT syndrome. Clin Case Rep. 2016:4(1):35-8.

16. Bjerregaard P, Jahangir A, Gussak I. Targeted therapy for short QT syndrome. Expert Opin Ther Targets. 2006;10(3):393-400

17. Duncan RS, McPate MJ, Ridley JM, Gao Z, James AF, Leishman DJ, Leaney JL, Witchel HJ, Hancox JC. Inhibition of the HERG potassium channel by the tricyclic antidepressant doxepin. Biochem Pharmacol. 2007;74(3):425-37.

18. Lu LX, Zhou W, Zhang X, Cao Q, Yu K, Zhu C. Short QT syndrome: a case report and review of literature. Resuscitation. 2006;71(1):115-21.

19. Harris L, McKenna WJ, Rowland E, Holt DW, Storey GC, Krikler DM. Side effects of long-term amiodarone therapy. Circulation. 1983;67(1):45-51.

20. Zemzemi N, Bernabeu MO, Saiz J, Cooper J, Pathmanathan P, Mirams GR, Pitt-Francis J, Rodriguez B. Computational assessment of drug-induced effects on the electrocardiogram: from ion channel to body surface potentials. Br J Pharmacol. 2013;168(3):718-33.

21. ten Tusscher KH, Panfilov AV. Alternans and spiral breakup in a human ventricular tissue model. Am J Physiol Heart Circ Physiol. 2006;291(3):H1088-100.

22. Priebe L, Beuckelmann DJ. Simulation study of cellular electric properties in heart failure. Circ Res. 1998;82(11):1206-23.

23. El Harchi A, McPate MJ, Zhang Y, Zhang H, Hancox JC. Action potential clamp and chloroquine sensitivity of mutant Kir2.1 channels responsible for variant 3 short QT syndrome. J Mol Cell Cardiol. 2009;47(5):743-7.

24. Brennan T, Fink M, Rodriguez B. Multiscale modelling of drug-induced effects on cardiac electrophysiological activity. Eur J Pharm Sci. 2009;36(1):62-77.

25. Kodama I, Kamiya K, Toyama J. Amiodarone: ionic and cellular mechanisms of action of the most promising class III agent. Am J Cardiol. 1999;84(9A):20R-8R.

26. Loewe A, Lutz Y, Wilhelms M, Sinnecker D, Barthel P, Scholz EP, Dossel O, Schmidt G, Seemann G. In-silico assessment of the dynamic effects of amiodarone and dronedarone on human atrial patho-electrophysiology. Europace. 2014;16(Suppl 4):iv30-8.

27. Kamiya K, Nishiyama A, Yasui K, Hojo M, Sanguinetti MC, Kodama I. Short- and long-term effects of amiodarone on the two components of cardiac delayed rectifier K(+) current. Circulation. 2001;103(9):1317-24.

28. Lalevee N, Nargeot J, Barrere-Lemaire S, Gautier P, Richard S. Effects of amiodarone and dronedarone on voltagedependent sodium current in human cardiomyocytes. J Cardiovasc Electrophysiol. 2003;14(8):885-90.

29. Gray DF, Mihailidou AS, Hansen PS, Buhagiar KA, Bewick NL, Rasmussen HH, Whalley DW. Amiodarone inhibits the $\mathrm{Na}(+)-\mathrm{K}+$ pump in rabbit cardiac myocytes after acute and chronic treatment. J Pharmacol Exp Ther. 1998;284(1):75-82.

30. Nishimura $\mathrm{M}$, Follmer $\mathrm{CH}$, Singer $\mathrm{DH}$. Amiodarone blocks calcium current in single guinea pig ventricular myocytes. J Pharmacol Exp Ther. 1989;251(2):650-9.

31. Watanabe Y, Kimura J. Acute inhibitory effect of dronedarone, a noniodinated benzofuran analogue of amiodarone, on $\mathrm{Na}+/ \mathrm{Ca} 2+$ exchange current in guinea pig cardiac ventricular myocytes. Naunyn Schmiedebergs Arch Pharmacol. 2008;377(4-6):371-6. 
32. Zankov DP, Ding WG, Matsuura H, Horie M. Open-state unblock characterizes acute inhibition of I potassium current by amiodarone in guinea pig ventricular myocytes. J Cardiovasc Electrophysiol. 2005;16(3):314-22.

33. O'Hara T, Virag L, Varro A, Rudy Y. Simulation of the undiseased human cardiac ventricular action potential: model formulation and experimental validation. PLoS Comput Biol. 2011;7(5):e1002061.

34. Rush S, Larsen H. A practical algorithm for solving dynamic membrane equations. IEEE Trans Biomed Eng. 1978;25(4):389-92.

35. Yan GX, Shimizu W, Antzelevitch C. Characteristics and distribution of M cells in arterially perfused canine left ventricular wedge preparations. Circulation. 1998;98(18):1921-7.

36. Adeniran I, MCPate MJ, Witchel HJ, Hancox JC, Zhang H. Increased vulnerability of human ventricle to re-entrant excitation in hERG-linked variant 1 short QT syndrome. PLoS Comput Biol. 2011;7(12):e1002313.

37. Zhang H, Kharche S, Holden AV, Hancox JC. Repolarisation and vulnerability to re-entry in the human heart with short QT syndrome arising from KCNQ1 mutation—a simulation study. Prog Biophys Mol Biol. 2008;96(1-3):112-31.

38. Weiss DL, Seemann G, Sachse FB, Dossel O. Modelling of short QT syndrome in a heterogeneous model of the human ventricular wall. Europace. 2005;7(Suppl 2):105-17.

39. Taggart P, Sutton PM, Opthof T, Coronel R, Trimlett R, Pugsley W, Kallis P. Inhomogeneous transmural conduction during early ischaemia in patients with coronary artery disease. J Mol Cell Cardiol. 2000;32(4):621-30.

40. Weingart $\mathrm{R}$. The actions of ouabain on intercellular coupling and conduction velocity in mammalian ventricular muscle. J Physiol. 1977;264(2):341-65.

41. Gima K, Rudy Y. Ionic current basis of electrocardiographic waveforms: a model study. Circ Res. 2002;90(8):889-96.

42. Drouin E, Charpentier F, Gauthier C, Laurent K, Le Marec H. Electrophysiologic characteristics of cells spanning the left ventricular wall of human heart: evidence for presence of M cells. J Am Coll Cardiol. 1995;26(1):185-92.

43. Xie F, Qu Z, Garfinkel A, Weiss JN. Electrical refractory period restitution and spiral wave reentry in simulated cardiac tissue. Am J Physiol Heart Circ Physiol. 2002;283(1):H448-60.

44. Yuan Y, Bai X, Luo C, Wang K, Zhang H. The virtual heart as a platform for screening drug cardiotoxicity. Br J Pharmacol. 2015;172(23):5531-47.

45. Mirams GR, Davies MR, Cui Y, Kohl P, Noble D. Application of cardiac electrophysiology simulations to pro-arrhythmic safety testing. Br J Pharmacol. 2012;167(5):932-45.

46. Sinha S, Pande A, Pandit R. Defibrillation via the elimination of spiral turbulence in a model for ventricular fibrillation. Phys Rev Lett. 2001;86(16):3678-81.

47. O'Hara T, Rudy Y. Quantitative comparison of cardiac ventricular myocyte electrophysiology and response to drugs in human and nonhuman species. Am J Physiol Heart Circ Physiol. 2012;302(5):H1023-30.

48. Trayanova NA. Whole-heart modeling: applications to cardiac electrophysiology and electromechanics. Circ Res. 2011:108(1):113-28

49. Cardona K, Trenor B, Molto G, Martinez M, Ferrero JM Jr, Starmer F, Saiz J. Exploring the role of pH in modulating the effects of lidocaine in virtual ischemic tissue. Am J Physiol Heart Circ Physiol. 2010;299(5):H1615-24.

Submit your next manuscript to BioMed Central and we will help you at every step:

- We accept pre-submission inquiries

- Our selector tool helps you to find the most relevant journal

- We provide round the clock customer support

- Convenient online submission

- Thorough peer review

- Inclusion in PubMed and all major indexing services

- Maximum visibility for your research

Submit your manuscript at www.biomedcentral.com/submit 